\title{
A variational problem arising in registration of diffusion tensor images *
}

\author{
Huan $\operatorname{Han}^{a}$ and Huan-Song Zhou ${ }^{b}$ \\ ${ }^{a}$ Wuhan Insitute of Physics and Mathematics, Chinese Academy of Sciences, \\ P.O. Box 71010, Wuhan, 430071, China \\ ${ }^{b}$ Department of Mathematics, Wuhan University of Technology, Wuhan 430070, China
}

\begin{abstract}
The existence of a global minimizer for a variational problem arising in registration of diffusion tensor images is proved, which ensures that there is a regular spatial transformation for the registration of diffusion tensor images.
\end{abstract}

Keywords: variational problem, minimizer, registration, diffusion tensor image. MSC2010: 97M10, 58E05, 49J20, 49J45, 49J35.

\section{Introduction}

Let $\Omega \subset \mathbb{R}^{3}$ be an open bounded domain with Liptschitz boundary $\partial \Omega$, and let $T$ and $D$ be two functions such that

$$
T: \Omega \rightarrow \mathbb{R}^{d} \quad \text { and } \quad D: \Omega \rightarrow \mathbb{R}^{d} \quad(d \geq 1) .
$$

By talking about the image registration, $T$ and $D$ are viewed as two images in a spatial domain $\Omega$, one is called the floating image(e.g. $T$ ) and the other is the target image (e.g. $D$ ). The registration of two images is to find a smooth and locally non-degenerate spatial transformation $h: \Omega \rightarrow \Omega$ such that the composition of $T$ and $h$, that is $T \circ h(x)=T(h(x))$, is close to $D$ in some sense, for example, find a $h$ such that the sum of squared errors in a suitable space reaches to its minimum. Note that the dimension $d$ in (1.1) of the space for the ranges of $T$ and $D$ varies with imaging modalities, for examples, images acquired by Magnetic Resonance Imaging(MRI) or Computed Tomography $(\mathrm{CT})$ are scalar valued with $d=1$, RGB images are vector valued with $d=3$, and the Diffusion Tensor Imaging(DTI) is matrix valued with $d=9$ (or $d=6$ if the matrix at each voxel of DTI is $3 \times 3$ symmetric positive).

In order to get a spatial transformation $h: \Omega \rightarrow \Omega$ with higher regularity, Dupuis, Grenander and Miller [5] improved the variational model proposed by Amit [2] for scalar image registration $(d=1)$, and they considered the following variational problem in a suitable space for $v$ :

$$
\hat{v}=\arg \min _{v} \int_{0}^{\tau}\|L v(\cdot, t)\|_{L^{2}(\Omega)}^{2} d t+\|T \circ h(\cdot)-D(\cdot)\|_{L^{2}(\Omega)}^{2},
$$

\footnotetext{
*Email: hanhuan11@mails.ucas.ac.cn (H. Han); hszhou@wipm.ac.cn (H. Zhou)

${ }^{\dagger}$ This work was supported by NSFC under grant No.11471331 and partially supported by National Center for Mathematics and Interdisciplinary Sciences.
} 
where $\tau>0$ is the time duration, "o" is the composition of two functions, and $L:\left[H_{0}^{3}(\Omega)\right]^{3} \rightarrow$ $\left[L^{2}(\Omega)\right]^{3}$ is a differential operator satisfying

$$
\|L v(\cdot, t)\|_{L^{2}(\Omega)}^{2}=\sum_{i=1}^{3} \int_{\Omega}\left|(L v)_{i}(x, t)\right|^{2} d x \geq c\|v(\cdot, t)\|_{\left[H_{0}^{3}(\Omega)\right]^{3}}^{2},
$$

for some $c>0$ and for all $v \in\left[H_{0}^{3}(\Omega)\right]^{3} \triangleq H_{0}^{3}(\Omega) \times H_{0}^{3}(\Omega) \times H_{0}^{3}(\Omega)$ with $H_{0}^{3}(\Omega)=W_{0}^{3,2}(\Omega)$. Clearly, $L=\sum_{i+j+k=3} \frac{\partial^{3}}{\partial x_{1}^{i} \partial x_{2}^{j} \partial x_{3}^{k}}$ satisfies (1.3). Furthermore, $v$ and $h$ are constrained by the following equation:

$$
\frac{d \eta(s ; t, x)}{d s} \triangleq \dot{\eta}(s ; t, x)=v(\eta(s ; t, x), s), \eta(t ; t, x)=x, h(x)=\eta(0 ; \tau, x), 0 \leq s, t \leq \tau,
$$

here $\eta(s ; t, x)$ means that a particle placed at $y=\eta(s ; t, x)$ at time $s$ is transformed to a point $x$ at time $t$ under the forcing term $v(x, t)$. In 5 , the authors gave a rigid mathematical proof on the existence of global minimizer, which validates the applications of model (1.2) in numerical simulations of scalar image registration. Based on [5], the large derivation principle(LDP) of the constraint equation (1.4) are concerned in 4. However, the model (1.2) does not work for registration of DTI images because each voxel of DTI image contains a $3 \times 3$ symmetric positive definite real matrix (i.e., diffusion tensor) and the orientation of diffusion tensors must be considered in making spatial transformation, which is much more complicated than that of scalar images. For this reason, there are two tensor reorientation strategies, the finite strain(FS) and the preservation principle direction(PPD), have been proposed by Alexander 1 , which are widely used for analyzing DTI data. Note that, for DTI images, both $T$ and $D$ are maps from $\Omega$ to the set of $3 \times 3$ Symmetric Positive Definite real matrixes $(S P D(3)$ in short), that is,

$$
T, D: \Omega \rightarrow S P D(3) \subset \mathbb{R}^{6} .
$$

In order to extend the variational model (1.2) to the case of registration of DTI images, based on FS reorientation strategy, Li et al. 8 introduced a new transformation operation " $\diamond$ " which is used to replace the usual composition operation "o" in (1.2). The operation " $\diamond$ " is given by

$$
T \diamond h(x)=R[T \circ h(x)] R^{T} \text { with } R=J_{x}^{T}\left(J_{x} J_{x}^{T}\right)^{-\frac{1}{2}} \text { and } J_{x}=\nabla_{x} h^{-1}(x),
$$

and the computation of $\left(J_{x} J_{x}^{T}\right)^{-\frac{1}{2}}$ is given in Appendix.

With the above definition and notations, the variational model proposed in 8 for the registration of DTI images can be formulated by

$$
\hat{v}=\arg \min _{v \in \mathcal{F}} H(v),
$$

where $H(v)=\int_{0}^{\tau}\|L v(\cdot, t)\|_{L^{2}(\Omega)}^{2} d t+\|T \diamond h(\cdot)-D(\cdot)\|_{L^{2}(\Omega)}^{2}, \tau$ and $L$ are the same as in (1.2), $h(x)$ is given by (1.4), $\mathcal{F}$ is defined by (1.10).

Remark 1.1. The constrain variational problem(1.7) and (1.4) is well defined, see Lemma 2.2. Moreover, $J_{x}$ defined in (1.6) satisfies

$$
J_{x}=\nabla_{x} h^{-1}(x)=\nabla_{x} \eta(\tau ; 0, x) .
$$

Note that here function $h^{-1}$ is well defined, see Remark 2.1. 
In fact, let $y=h(x)=\eta(0 ; \tau, x)$, then by definition of $\eta(s ; t, x)$, we know $x=h^{-1}(y)=$ $\eta(\tau ; 0, y)$. Hence, for any particle at position $y$ of the moving image at time 0 , the position at time $\tau$ of this particle is $x$. By [1, Section III], we know $J_{y}=\nabla_{y} x$, that is,

$$
J_{y}=\nabla_{y} \eta(\tau ; 0, y)
$$

The authors of $[8$ designed an efficient numerically algorithm for registration of DTI images based on (1.7) with $L \equiv 0$. If $L \neq 0$, the situation becomes much more complicated. To ensure the model (1.7) can be well used in developing an optimal algorithm of DTI registration, it is important to know mathematically the solvability of (1.7). To the authors' knowledge, there seems no any results on the existence of global minimizer for variational problem (1.7). Motivated by [5, the aim of this paper is to give a rigid mathematical proof on the existence.

Before giving our main result, we introduce some notations.

For a given $\tau>0$ and $u(x, t): \Omega \times[0, \tau] \rightarrow \mathbb{R}^{3}$, let $L$ be a linear differential operator given by (1.3), we define

$$
\mathcal{F} \triangleq\left\{u(x, t): u(x, t) \in\left[H_{0}^{3}(\Omega)\right]^{3} \text { for any } t \in[0, \tau] \text { and } \int_{0}^{\tau}\|L u(\cdot, t)\|_{L^{2}(\Omega)}^{2} d t<+\infty\right\},
$$

endowing with the following inner product and norm

$$
(u, v)_{\mathcal{F}}=\int_{0}^{\tau} \int_{\Omega} L u(x, t) \cdot L v(x, t) d x d t \text { and }\|u\|_{\mathcal{F}}^{2}=\int_{0}^{\tau}\|L u(\cdot, t)\|_{L^{2}(\Omega)}^{2} d t,
$$

and $\mathcal{F}$ is a separable Hilbert space associated with the above norm.

Throughout the paper, the norm of a matrix $A(x)=\left(a_{i j}(x)\right)_{n \times m}$ is defined by

$$
\|A(x)\|=\sum_{i=1}^{n} \sum_{j=1}^{m}\left|a_{i j}(x)\right|
$$

We say a matrix $A(x)$ is continuous at $x \in \Omega$ if each element $a_{i j}(x)$ of $A(x)$ is continuous at $x$. Particularly, if $m=1$, the matrix becomes an $n \times 1$ vector which is usually denoted $v(x)=\left(v_{1}(x), v_{2}(x), \cdots, v_{n}(x)\right)^{T}$ with the norm $\|v(x)\|=\sum_{i=1}^{n}\left|v_{i}(x)\right|$.

Based on the above definitions and notations, our main result can be stated as follows:

Theorem 1.1. For $\Omega$ being given in (1.1), let $T$ and $D$ be the two maps defined by (1.5), and let the set $\triangle_{T} \triangleq\{x \in \Omega: T(\cdot)$ is discontinuous at $x\}$ be a set of measure zero. If $L:\left[H_{0}^{3}(\Omega)\right]^{3} \rightarrow$ $\left[L^{2}(\Omega)\right]^{3}$ is a linear differential operator and satisfies (1.3) and

$$
\max _{x \in \Omega}\|T(x)\|<+\infty, J=\max _{x \in \Omega}\|T(x)-D(x)\|^{2}<+\infty,
$$

then the variational problem (1.7) has a global minimizer $\hat{v} \in \mathcal{F}$, which induces a deformation $\hat{h}(x) \in\left[C^{1, \frac{1}{2}}(\Omega)\right]^{3}$ from $\Omega$ to $\Omega$. Moreover, the derivative of $\hat{h}(x)$ satisfies [2.6).

\section{Preliminary results}

In this section, we show some lemmas which are required in proving Theorem 1.1 . 
Lemma 2.1. For any $f=\left(f_{1}, f_{2}, f_{3}\right)^{T} \in\left[H_{0}^{3}(\Omega)\right]^{3}$, there exists $K>0$ such that

(i) $\|f(x)-f(y)\| \leq K\|L f\|_{L^{2}(\Omega)}\|x-y\|, \forall x, y \in \Omega$.

(ii) $\|\nabla f(x)-\nabla f(y)\| \leq K\|L f\|_{L^{2}(\Omega)}\|x-y\|^{\frac{1}{2}}, \forall x, y \in \Omega$, where $\nabla f=\left(f_{i j}\right)_{3 \times 3}$ with $f_{i j}=\frac{\partial f_{i}}{\partial x_{j}}(i, j=1,2,3)$.

Proof: (i) By Taylor's formula, there holds

$$
\|f(x)-f(y)\|=C\left\|\nabla f(\zeta) \cdot(x-y)^{T}\right\|
$$

Note that $H^{3}(\Omega) \hookrightarrow C^{1, \frac{1}{2}}(\Omega)$, then

$$
\|f(x)-f(y)\| \leq C\|\nabla f(\zeta)\|\|x-y\| \leq \tilde{C}\|f\|_{C^{1, \frac{1}{2}}(\Omega)}\|x-y\| \leq K\|L f\|_{L^{2}(\Omega)}\|x-y\| .
$$

(ii) Note that $H^{3}(\Omega) \hookrightarrow C^{1, \frac{1}{2}}(\Omega)$. Then,

$$
\frac{\left\|\nabla f_{i}(x)-\nabla f_{i}(y)\right\|}{\|x-y\|^{\frac{1}{2}}} \leq\left\|f_{i}\right\|_{C^{1, \frac{1}{2}}(\Omega)} \leq C\left\|f_{i}\right\|_{H_{0}^{3}(\Omega)} \leq \tilde{K}\|L f\|_{L^{2}(\Omega)}
$$

That implies,

$$
\left\|\nabla f_{i}(x)-\nabla f_{i}(y)\right\| \leq \tilde{K}\|L f\|_{L^{2}(\Omega)}\|x-y\|^{\frac{1}{2}} .
$$

Hence, we obtain that

$\|\nabla f(x)-\nabla f(y)\|=\sum_{i=1}^{3}\left\|\nabla f_{i}(x)-\nabla f_{i}(y)\right\| \leq K\|L f\|_{L^{2}(\Omega)}\|x-y\|^{\frac{1}{2}}$.

By (1.7), we know that $H(v)$ is essentially a functional of $v$ and $\eta$, where $v$ and $\eta$ satisfy (1.4). If we write $H(v)$ as a functional about $v$, then the existence and uniqueness for the solutions of (1.4) should be known. Otherwise, the definition of (1.7) will be ambiguous if one $v$ produces two or more $\eta$ by (1.4). So, the following results on the ODE problem (1.4) imply that the variational problem (1.7) and (1.4) is well defined.

Lemma 2.2. Let $v \in \mathcal{F}$ and $\|v\|_{\mathcal{F}}^{2}<+\infty$. If $\left.v(\cdot, t)\right|_{\mathbb{R}^{3} \backslash \Omega}=0$ for each $t \in[0, \tau]$, then there exists a unique solution $\eta(s ; t, x) \in C([0, \tau], \bar{\Omega})$ of [1.4) for $s \in[0, \tau]$ and $x \in \bar{\Omega}$.

Proof: First, we choose a constant $M$ large enough such that $\|v\|_{\mathcal{F}}^{2} \leq M$. For $x \in \bar{\Omega}$ and $s \in[0, \tau]$, we define

$$
\Gamma_{s}(\phi)=x+\int_{t}^{s} v(\phi(r), r) d r \text { for } \phi \in C\left([0, \tau], \mathbb{R}^{3}\right)
$$

Then, we have

$$
\begin{aligned}
\left\|\Gamma_{s}(\phi)\right\| \leq & \|x\|+\left\|\int_{t}^{s} v(\phi(r), r) d r\right\| \leq\|x\|+\left|\int_{0}^{\tau}\|v(\phi(r), r)\| d r\right| \\
& \leq\|x\|+\left|\int_{0}^{\tau}\|v(\cdot, r)\|_{C(\Omega)} d r\right| \leq\|x\|+c_{1}\left|\int_{0}^{\tau}\|v(\cdot, r)\|_{H^{3}(\Omega)} d r\right| \\
& \leq\|x\|+c\left|\int_{0}^{\tau}\|L v(\cdot, r)\|_{L^{2}(\Omega)} d r\right| \leq\|x\|+c \tau^{\frac{1}{2}}\left(\int_{0}^{\tau}\|L v(\cdot, r)\|_{L^{2}(\Omega)}^{2} d r\right)^{\frac{1}{2}} \\
& \leq E+c(M \tau)^{\frac{1}{2}} \triangleq \bar{M},
\end{aligned}
$$


where $E=\sup \{\|x\|: x \in \Omega\}$.

Now we claim that the mapping $\Gamma_{s}$ from $C\left([0, \tau], \mathbb{R}^{3}\right)$ to $C\left([0, \tau], \mathbb{R}^{3}\right)$ is continuous. In fact, by the definition of $\Gamma_{s}$ and Lemma 2.1, we obtain that

$$
\begin{aligned}
& \left\|\Gamma_{s}\left(\phi_{1}\right)-\Gamma_{s}\left(\phi_{2}\right)\right\| \leq\left|\int_{t}^{s}\left\|v\left(\phi_{1}(r), r\right)-v\left(\phi_{2}(r), r\right)\right\| d r\right| \\
\leq & K\left|\int_{t}^{s}\|L v(\cdot, r)\|_{L^{2}(\Omega)}\left\|\phi_{1}(r)-\phi_{2}(r)\right\| d r\right| \leq K\left|\int_{t}^{s}\|L v(\cdot, r)\|_{L^{2}(\Omega)} d r\right|\left\|\phi_{1}-\phi_{2}\right\|_{C([0, \tau], \bar{\Omega})} \\
\leq & K \tau^{\frac{1}{2}}\left(\int_{0}^{\tau}\|L v(\cdot, r)\|_{L^{2}(\Omega)}^{2} d r\right)^{\frac{1}{2}}\left\|\phi_{1}-\phi_{2}\right\|_{C([0, \tau], \bar{\Omega})} \leq K(M \tau)^{\frac{1}{2}}\left\|\phi_{1}-\phi_{2}\right\|_{C([0, \tau], \bar{\Omega})} .
\end{aligned}
$$

Hence,

$$
\left\|\Gamma_{s}\left(\phi_{1}\right)-\Gamma_{s}\left(\phi_{2}\right)\right\|_{C([0, \tau], \bar{\Omega})} \leq K(M \tau)^{\frac{1}{2}}\left\|\phi_{1}-\phi_{2}\right\|_{C([0, \tau], \bar{\Omega})},
$$

and the claim is proved.

Next, we show that $\Gamma_{s}$ from $C\left([0, \tau], \mathbb{R}^{3}\right)$ to $C\left([0, \tau], \mathbb{R}^{3}\right)$ is compact. In fact, for $0 \leq t_{1}<$ $t_{2} \leq \tau$, we see that

$$
\begin{aligned}
\left\|\Gamma_{t_{1}}(\phi)-\Gamma_{t_{2}}(\phi)\right\| & =\left\|\int_{t_{1}}^{t_{2}} v(\phi(r), r) d r\right\| \leq\left|t_{2}-t_{1}\right|^{\frac{1}{2}}\left(\int_{t_{1}}^{t_{2}}\|v(\phi(r), r)\|^{2} d r\right)^{\frac{1}{2}} \\
& \leq\left|t_{2}-t_{1}\right|^{\frac{1}{2}}\left(\int_{t_{1}}^{t_{2}}\|v(\cdot, r)\|_{C^{1, \frac{1}{2}}(\Omega)}^{2} d r\right)^{\frac{1}{2}} \leq C\left|t_{2}-t_{1}\right|^{\frac{1}{2}}\left(\int_{t_{1}}^{t_{2}}\|v(\cdot, r)\|_{H_{0}^{3}(\Omega)}^{2} d r\right)^{\frac{1}{2}} \\
& \leq K\left|t_{2}-t_{1}\right|^{\frac{1}{2}}\left(\int_{t_{1}}^{t_{2}}\|L v(\cdot, r)\|_{L^{2}(\Omega)}^{2} d r\right)^{\frac{1}{2}} \leq K\left|t_{2}-t_{1}\right|^{\frac{1}{2}}\left(\int_{0}^{\tau}\|L v(\cdot, r)\|_{L^{2}(\Omega)}^{2} d r\right)^{\frac{1}{2}} \\
& \leq K M^{\frac{1}{2}}\left|t_{2}-t_{1}\right|^{\frac{1}{2}},
\end{aligned}
$$

this means that $\left\{\Gamma_{s}(\phi), \phi \in C\left([0, \tau], \mathbb{R}^{3}\right)\right\}$ is equicontinuous for $s \in[0, \tau]$. Then the ArzelaAscoli Theorem $\left[9\right.$ shows that $\left\{\Gamma(\phi): \phi \in C\left([0, \tau], \mathbb{R}^{3}\right)\right\}$ is relative compact, which means that any bounded sequence has as convergent subsequence. Hence, the mapping $\Gamma_{s}: C\left([0, \tau], \mathbb{R}^{3}\right) \rightarrow$ $C\left([0, \tau], \mathbb{R}^{3}\right)$ is compact. On the other hand, the set

$$
\Lambda \triangleq\left\{\phi \in C\left([0, \tau], \mathbb{R}^{3}\right): \phi=\lambda \Gamma_{s}(\phi) \text { for some } 0 \leq \lambda \leq 1\right\}
$$

is not empty and bounded. In fact, it follows from (2.1) that

$$
\begin{aligned}
\left\|\lambda \Gamma_{s}\left(\phi_{1}\right)-\lambda \Gamma_{s}\left(\phi_{2}\right)\right\| & =\lambda\left\|\int_{t}^{s}\left[v\left(\phi_{1}(r), r\right)-v\left(\phi_{1}(r), r\right)\right] d r\right\| \leq \lambda\left|\int_{0}^{\tau} K\|L v(\cdot, r)\|_{L^{2}(\Omega)}\left\|\phi_{1}(r)-\phi_{2}(r)\right\| d r\right| \\
& \leq \lambda K \int_{0}^{\tau}\|L v(\cdot, r)\|_{L^{2}(\Omega)} d r\left\|\phi_{1}(\cdot)-\phi_{2}(\cdot)\right\|_{C\left([0, \tau], \mathbb{R}^{3}\right)} \\
& \leq \lambda K \tau^{\frac{1}{2}}\left(\int_{0}^{\tau}\|L v(\cdot, r)\|_{L^{2}(\Omega)}^{2} d r\right)^{\frac{1}{2}}\left\|\phi_{1}(\cdot)-\phi_{2}(\cdot)\right\|_{C\left([0, \tau], \mathbb{R}^{3}\right)} \\
& \leq \lambda K(M \tau)^{\frac{1}{2}}\left\|\phi_{1}(\cdot)-\phi_{2}(\cdot)\right\|_{C\left([0, \tau], \mathbb{R}^{3}\right)}
\end{aligned}
$$

this implies that $\lambda \Gamma_{s}$ is a strict contraction if $\lambda \in\left(0, \frac{1}{K(M \tau)^{\frac{1}{2}}}\right)$, and the Banach's fixed point theorem shows that $\Lambda \neq \emptyset$. Furthermore, for any $\tilde{\phi} \in C\left([0, \tau], \mathbb{R}^{3}\right)$ satisfies

$$
\tilde{\phi}=\lambda \Gamma_{s}(\tilde{\phi}), 0 \leq \lambda \leq 1,
$$


it follows from (2.2) that $\|\tilde{\phi}\|=\left\|\lambda \Gamma_{s}(\tilde{\phi})\right\| \leq \lambda\left\|\Gamma_{s}(\tilde{\phi})\right\| \leq \widetilde{M}$, that is, the set $\Lambda$ is bounded, and the claim is proved.

With the above facts, the Schaefer's Fixed Point Theorem[6, Theorem 4 in Section 9.2] shows that $\Gamma_{s}$ has a fixed point $\phi(s)$ such that

$$
\phi(s)=\Gamma_{s}(\phi)=x+\int_{t}^{s} v(\phi(r), r) d r \in C([0, \tau], \bar{\Omega}) .
$$

Define $\eta(s ; t, x)=\phi(s)$, then we know (1.4) has a solution.

Finally, we prove the uniqueness of the solution of (1.4).

Assume there $\eta_{1}(s ; t, x), \eta_{2}(s ; t, x)$ are two different solutions of (1.4), then

$$
\begin{aligned}
\left\|\eta_{1}(s ; t, x)-\eta_{2}(s ; t, x)\right\| & =\| \int_{t}^{s} v\left(\eta_{1}(r ; t, x), r\right)-v\left(\eta_{2}(r ; t, x), r\right) d r|| \\
& \leq\left|\int_{t}^{s}\left\|v\left(\eta_{1}(r ; t, x), r\right)-v\left(\eta_{2}(r ; t, x), r\right)\right\| d r\right| \\
& \leq\left|\int_{t}^{s} K\|L v\|_{L^{2}(\Omega)}\left\|\eta_{1}(r ; t, x)-\eta_{2}(r ; t, x)\right\| d r\right| .
\end{aligned}
$$

By Grownwall inequality[10, Lemma 1.1], we know that

$$
\left\|\eta_{1}(s ; t, x)-\eta_{2}(s ; t, x)\right\|=0 .
$$

Hence, $\eta_{1}(s ; t, x) \equiv \eta_{2}(s ; t, x)$.

Our following lemma shows that $J_{x}$ is not singular.

Lemma 2.3. Let $v \in \mathcal{F}$ and the condition (1.3) be satisfied. Then

(i) For any $t \in[0, \tau], s \in[0, \tau]$ and $x \in \bar{\Omega}, \eta(s ; t, x)$ is differentiable with respect to (w.r.t. in short) $x$ and the derivative $\nabla_{x} \eta(s ; t, x) \triangleq \Theta(s ; t, x)$ satisfies

$$
\left\{\begin{array}{l}
\dot{\Theta}(s ; t, x)=\nabla_{\eta} v(\eta(s ; t, x), s) \Theta(s ; t, x), \\
\Theta(t ; t, x)=I=\left(\delta_{i j}\right)_{3 \times 3}
\end{array}\right.
$$

where $\delta_{i j}=0$ if $i \neq j, \delta_{i j}=1$ if $i=j$, and $\nabla_{\eta} v(\eta(s ; t, x), s)=\left(\frac{\partial v_{i}}{\partial \eta_{j}}(\eta(s ; t, x), s)\right)_{3 \times 3}$.

(ii) The determinant of $\Theta(s ; t, x)$ is equal to

$$
\operatorname{det}(\Theta(s ; t, x))=e^{\int_{t}^{s} \sum_{i=1}^{3} v_{i, \eta_{i}}(\eta(s ; t, x), s) d s} .
$$

Proof: (i) By (1.3) and the fact that $H_{0}^{3}(\Omega) \hookrightarrow C^{1, \frac{1}{2}}(\Omega)$, we know

$$
\dot{\eta}(s ; t, x)=v(\eta(s ; t, x), s), \quad \eta(t ; t, x)=x,
$$

and $v(\eta(s ; t, x), s)$ is differentiable w.r.t $\eta(s ; t, x)$ and $\eta(s ; t, x)$ is differentiable w.r.t. $x$ (cf. [12]).

Differentiating the first equation of (2.7) w.r.t. $x$, we have

$$
\frac{d}{d s} \nabla_{x} \eta(s ; t, x)=\nabla_{x}(v(\eta(s ; t, x), s))=\nabla_{\eta} v(\eta(s ; t, x), s) \cdot \nabla_{x} \eta(s ; t, x),
$$


that is,

$$
\dot{\Theta}(s ; t, x)=\nabla_{\eta} v(\eta(s ; t, x), s) \cdot \Theta(s ; t, x) .
$$

If we differentiate the second equation of (2.7) w.r.t. $x$, which gives

$$
\Theta(t ; t, x)=I,
$$

that is, the second equation of (2.6). So, part (i) is proved.

(ii) Let $\theta_{i j}, v_{i j}$ be the element at the $i t h$ row and the $j t h$ column of $\Theta(s ; t, x), \nabla_{\eta} v(\eta(s ; t, x), s)$ respectively, then we obtain that

$$
\frac{d \operatorname{det}(\Theta(s ; t, x))}{d s}=\left(v_{11}+v_{22}+v_{33}\right) \operatorname{det}(\Theta(s ; t, x)) .
$$

Hence,

$\operatorname{det}(\Theta(s ; t, x))=\operatorname{det}(I) e^{\int_{t}^{s} v_{11}+v_{22}+v_{33} d s}=e^{\int_{t}^{s} \sum_{i=1}^{3} v_{i, \eta_{i}}(\eta(s ; t, x), s) d s}$.

Remark 2.1. Let $t=\tau$ and $s=0$, by Lemma 2.3(ii), there holds

$$
\operatorname{det}(\nabla h(x))=\operatorname{det}(\Theta(0 ; \tau, x))=e^{-\int_{0}^{\tau} \sum_{i=1}^{3} v_{i, \eta_{i}}(\eta(s ; t, x), s) d s} \neq 0,
$$

for all $x \in \Omega$. By Inverse Function Theorem[5, Theorem 7 in Appendix], (2.10) ensures the existence of $h^{-1}$.

Lemma 2.4. For $\Theta_{n}(n=1,2,3, \cdots), \Theta \in S P D(3)$, let $\lambda_{n}^{(1)} \geq \lambda_{n}^{(2)} \geq \lambda_{n}^{(3)}>0$ and $\lambda^{(1)} \geq \lambda^{(2)} \geq$ $\lambda^{(3)}>0$ be eigenvalues of $\Theta_{n}$ and $\Theta$, respectively. If $\Theta_{n} \stackrel{n}{\rightarrow} \Theta$ (i.e. each element of $\Theta_{n}$ converges to that of $\Theta)$, then $\lambda_{n}^{(i)} \stackrel{n}{\rightarrow} \lambda^{(i)}(i=1,2,3)$.

Proof: Define $\Theta_{n}=\left(\theta_{i j}^{n}\right)_{3 \times 3}, \Theta=\left(\theta_{i j}\right)_{3 \times 3}$. Let

$$
\operatorname{det}\left(\tilde{\lambda} I-\Theta_{n}\right)=0 \text { and } \operatorname{det}(\lambda I-\Theta)=0 .
$$

Since $\Theta_{n}, \Theta \in S P D(3)$, we know that the equations of (2.11) have the following form

$$
\tilde{\lambda}^{3}+p_{n} \tilde{\lambda}^{2}+q_{n} \tilde{\lambda}+r_{n}=0, \lambda^{3}+p \lambda^{2}+q \lambda+r=0
$$

where $p_{n}=-\left(\theta_{11}^{n}+\theta_{22}^{n}+\theta_{33}^{n}\right), q_{n}=\theta_{11}^{n} \theta_{22}^{n}-\theta_{12}^{n} \theta_{21}^{n}+\theta_{11}^{n} \theta_{33}^{n}-\theta_{13}^{n} \theta_{31}^{n}+\theta_{22}^{n} \theta_{33}^{n}-\theta_{23}^{n} \theta_{32}^{n}, r_{n}=$ $-\theta_{11}^{n} \theta_{22}^{n} \theta_{33}^{n}+\theta_{11}^{n} \theta_{23}^{n} \theta_{32}^{n}+\theta_{12}^{n} \theta_{21}^{n} \theta_{33}^{n}-\theta_{12}^{n} \theta_{23}^{n} \theta_{31}^{n}-\theta_{13}^{n} \theta_{21}^{n} \theta_{32}^{n}+\theta_{13}^{n} \theta_{22}^{n} \theta_{31}^{n} \cdot p, q, r$ are of the same form as $p_{n}, q_{n}, r_{n}$, but $\theta_{i j}^{n}$ is replaced by $\theta_{i j}$. Therefore,

$$
p_{n} \stackrel{n}{\rightarrow} p, q_{n} \stackrel{n}{\rightarrow} q, r_{n} \stackrel{n}{\rightarrow} r .
$$

Since $\lambda_{n}^{(1)} \geq \lambda_{n}^{(2)} \geq \lambda_{n}^{(3)}>0$ and $\lambda^{(1)} \geq \lambda^{(2)} \geq \lambda^{(3)}>0$ are eigenvalues of $\Theta_{n}$ and $\Theta$ respectively, it follows from (2.12) that

$$
\left(\tilde{\lambda}-\lambda_{n}^{(1)}\right)\left(\tilde{\lambda}-\lambda_{n}^{(2)}\right)\left(\tilde{\lambda}-\lambda_{n}^{(3)}\right)=0, \quad\left(\lambda-\lambda^{(1)}\right)\left(\lambda-\lambda^{(2)}\right)\left(\lambda-\lambda^{(3)}\right)=0,
$$

and $p_{n}=-\left(\lambda_{n}^{(1)}+\lambda_{n}^{(2)}+\lambda_{n}^{(3)}\right), q_{n}=\lambda_{n}^{(1)} \lambda_{n}^{(2)}+\lambda_{n}^{(1)} \lambda_{n}^{(3)}+\lambda_{n}^{(2)} \lambda_{n}^{(3)}, r_{n}=-\lambda_{n}^{(1)} \lambda_{n}^{(2)} \lambda_{n}^{(3)}, p=$ $-\left(\lambda^{(1)}+\lambda^{(2)}+\lambda^{(3)}\right), q=\lambda^{(1)} \lambda^{(2)}+\lambda^{(1)} \lambda^{(3)}+\lambda^{(2)} \lambda^{(3)}, r=-\lambda^{(1)} \lambda^{(2)} \lambda^{(3)}$. 
Furthermore, for $i=1,2,3$, using (2.12) we see that

$$
\begin{aligned}
& \left(\lambda^{(i)}-\lambda_{n}^{(1)}\right)\left(\lambda^{(i)}-\lambda_{n}^{(2)}\right)\left(\lambda^{(i)}-\lambda_{n}^{(3)}\right)=\left(\lambda^{(i)}\right)^{3}+p_{n}\left(\lambda^{(i)}\right)^{2}+q_{n} \lambda^{(i)}+r_{n} \\
= & {\left[\left(\lambda^{(i)}\right)^{3}+p\left(\lambda^{(i)}\right)^{2}+q \lambda^{(i)}+r\right]+\left(p_{n}-p\right)\left(\lambda^{(i)}\right)^{2}+\left(q_{n}-q\right) \lambda^{(i)}+r_{n}-r } \\
= & \left(p_{n}-p\right)\left(\lambda^{(i)}\right)^{2}+\left(q_{n}-q\right) \lambda^{(i)}+r_{n}-r \stackrel{n}{\rightarrow} 0 .
\end{aligned}
$$

Then, either $\lambda^{(i)}-\lambda_{n}^{(1)} \stackrel{n}{\rightarrow} 0$, or $\lambda^{(i)}-\lambda_{n}^{(2)} \stackrel{n}{\rightarrow} 0$, or $\lambda^{(i)}-\lambda_{n}^{(3)} \stackrel{n}{\rightarrow} 0$. Note that $\lambda_{n}^{(1)}+\lambda_{n}^{(2)}+\lambda_{n}^{(3)}=$ $p_{n} \stackrel{n}{\rightarrow} p=\lambda^{(1)}+\lambda^{(2)}+\lambda^{(3)}, \lambda_{n}^{(1)} \lambda_{n}^{(2)}+\lambda_{n}^{(1)} \lambda_{n}^{(3)}+\lambda_{n}^{(2)} \lambda_{n}^{(3)}=q_{n} \stackrel{n}{\rightarrow} q=\lambda^{(1)} \lambda^{(2)}+\lambda^{(1)} \lambda^{(3)}+\lambda^{(2)} \lambda^{(3)}$ and $\lambda_{n}^{(1)} \lambda_{n}^{(2)} \lambda_{n}^{(3)}=-r_{n} \stackrel{n}{\rightarrow}-r=\lambda^{(1)} \lambda^{(2)} \lambda^{(3)}$. Therefore, $\lambda_{n}^{(i)} \stackrel{n}{\rightarrow} \lambda^{(i)}(i=1,2,3)$.

Lemma 2.5. Let $C_{m}, B_{m}$ be $3 \times 3$ matrixes with $\left|\operatorname{det}\left(B_{m}\right)\right| \geq M_{0}>0$ and $\left\|B_{m}\right\| \leq M_{1}<+\infty$. If $C_{m} B_{m} \stackrel{m}{\longrightarrow} O_{3 \times 3}$, then $C_{m} \stackrel{m}{\longrightarrow} O_{3 \times 3}$, where $O_{3 \times 3}$ denotes the $3 \times 3$ zero matrix (i.e. all elements of the matrix are 0$)$.

Proof: Let $C_{m}=\left(c_{i j}^{m}\right)_{3 \times 3}$ and $B_{m}=\left(b_{i j}^{m}\right)_{3 \times 3}$. Since $C_{m} B_{m} \stackrel{m}{\longrightarrow} O_{3 \times 3}, B_{m}^{T} C_{m}^{T} \stackrel{m}{\longrightarrow} O_{3 \times 3}$, that is,

$$
B_{m}^{T} C_{m}^{i} \stackrel{m}{\longrightarrow} O_{3 \times 1} \quad i=1,2,3,
$$

where $C_{m}^{i}=\left(c_{i 1}^{m}, c_{i 2}^{m}, c_{i 3}^{m}\right)^{T}$.

Therefore, there exists $\varepsilon_{i 1}^{m} \stackrel{m}{\longrightarrow} 0, \varepsilon_{i 2}^{m} \stackrel{m}{\longrightarrow} 0, \varepsilon_{i 3}^{m} \stackrel{m}{\longrightarrow} 0$ such that

$$
B_{m}^{T} C_{m}^{i}=\left(\varepsilon_{i 1}^{m}, \varepsilon_{i 2}^{m}, \varepsilon_{i 3}^{m}\right)^{T} \quad i=1,2,3 .
$$

By Cramer's Rule[11, Section 5.3], we obtain that

$$
\begin{aligned}
& c_{i 1}^{m}=\frac{\varepsilon_{i 1}^{m} B_{m}^{11 *}+\varepsilon_{i 2}^{m} B_{m}^{12 *}+\varepsilon_{i 3}^{m} B_{m}^{13 *}}{\operatorname{det}\left(B_{m}\right)}, \\
& c_{i 2}^{m}=\frac{\varepsilon_{i 1}^{m} B_{m}^{21 *}+\varepsilon_{i 2}^{m} B_{m}^{22 *}+\varepsilon_{i 3}^{m} B_{m}^{23 *}}{\operatorname{det}\left(B_{m}\right)}, \\
& c_{i 3}^{m}=\frac{\varepsilon_{i 1}^{m} B_{m}^{31 *}+\varepsilon_{i 2}^{m} B_{m}^{32 *}+\varepsilon_{i 3}^{m} B_{m}^{33 *}}{\operatorname{det}\left(B_{m}\right)},
\end{aligned}
$$

where $B_{m}^{i j *}$ is the cofactor of $i t h$ row and $j t h$ column element of $B_{m}$.

Since $\left|B_{m}^{i j *}\right| \leq 2\left\|B_{m}\right\|^{2} \leq 2 M_{1}$, it follows from (2.18), (2.19) and (2.20) that

$$
\left|c_{i j}^{m}\right| \leq \frac{2\left\|B_{m}\right\|^{2}\left(\left|\varepsilon_{i 1}^{m}\right|+\left|\varepsilon_{i 2}^{m}\right|+\left|\varepsilon_{i 3}^{m}\right|\right)}{\left|\operatorname{det}\left(B_{m}\right)\right|} \leq \frac{2 M_{1}^{2}\left(\left|\varepsilon_{i 1}^{m}\right|+\left|\varepsilon_{i 2}^{m}\right|+\left|\varepsilon_{i 3}^{m}\right|\right)}{M_{0}} \stackrel{m}{\longrightarrow} 0 \quad i, j=1,2,3 .
$$

Therefore, $C_{m} \stackrel{m}{\longrightarrow} O_{3 \times 3}$.

Lemma 2.6. Let $\left\{v_{n} \in \mathbb{R}^{3}\right\}$ be any sequence of $\mathcal{F}$ and

$$
\left\|v_{n}\right\|_{\mathcal{F}}^{2}=\int_{0}^{\tau}\left\|L v_{n}(\cdot, t)\right\|_{L^{2}(\Omega)}^{2} d t \leq M<+\infty
$$

and $\left.v_{n}(\cdot, t)\right|_{\partial \Omega}=0$ for each $t \in[0, \tau]$, then 
(i) There exists a subsequence $\left\{v_{n_{k}}\right\}$ of $\left\{v_{n}\right\}$ and some $v \in \mathcal{F}$ such that $v_{n_{k}} \stackrel{k}{\rightarrow} v$ weakly in $\mathcal{F}$. Moreover,

$$
M \geq \lim _{n_{k} \rightarrow \infty} \inf \int_{0}^{\tau}\left\|L v_{n_{k}}(\cdot, t)\right\|_{L^{2}(\Omega)}^{2} d t \geq \int_{0}^{\tau}\|L v(\cdot, t)\|_{L^{2}(\Omega)}^{2} d t
$$

(ii) Let $\left\{v_{n_{k}}\right\}$ be the subsequence obtained in (i) and let $v$ be its weak limit. For any given $t \in[0, \tau]$, consider the following equations,

$$
\dot{\eta}_{n_{k}}(s ; t, x)=v_{n_{k}}\left(\eta_{n_{k}}(s ; t, x), s\right) \text { with } \eta_{n_{k}}(t ; t, x)=x,
$$

and

$$
\dot{\eta}(s ; t, x)=v(\eta(s ; t, x), s) \text { with } \eta(t ; t, x)=x,
$$

then, these two equations have unique solution in $C([0, \tau]: \bar{\Omega})$ for all $x \in \Omega$. Moreover, for each $s \in[0, \tau], \eta_{n_{k}}(s ; t, x) \in C^{1, \frac{1}{2}}(\Omega), \eta(s ; t, x) \in C^{1, \frac{1}{2}}(\Omega)$ with $\eta_{n_{k}}(s ; t, x) \stackrel{k}{\rightarrow} \eta(s ; t, x)$ uniformly on $[0, \tau]$, and

$$
h_{n_{k}}(x)=\eta_{n_{k}}(0 ; \tau, x) \stackrel{k}{\rightarrow} \eta(0 ; \tau, x)=h(x) \text { for } \forall x \in \Omega .
$$

(iii) Let $\Theta_{n_{k}}(s ; 0, x)=\nabla_{x} \eta_{n_{k}}(s ; 0, x)$ and $\Theta(s ; 0, x)=\nabla_{x} \eta(s ; 0, x)$, where at $t=0 \eta_{n_{k}}(s ; 0, x)$ and $\eta(s ; 0, x)$ are solutions of 2.24) and (2.25), respectively. Then $\Theta_{n_{k}}(s ; 0, x) \stackrel{k}{\rightarrow} \Theta(s ; 0, x)$ in the norm (1.12) for all $(x, s) \in \Omega \times[0, \tau]$.

(iv) Let $R_{n_{k}}=\Theta_{n_{k}}\left(\Theta_{n_{k}} \Theta_{n_{k}}^{T}\right)^{-\frac{1}{2}}$ and $R=\Theta\left(\Theta \Theta^{T}\right)^{-\frac{1}{2}}$, here we simply denote $\Theta_{n_{k}}(s ; 0, x)$ and $\Theta(s ; 0, x)$ by $\Theta_{n_{k}}$ and $\Theta$, respectively. Then $R_{n_{k}} \stackrel{k}{\rightarrow} R$ in the norm (1.12) for all $(x, s) \in \Omega \times[0, \tau]$.

Proof: (i) and (ii) can be proved by the ideas of [5] and the Sobolev inequalities[6, Theorem 6 in Section 5.6]. Here, we focus on the proofs of (iii) and (iv).

(iii) By Lemma 2.3, we know that $\Theta_{n_{k}}(s ; 0, x)$ and $\Theta(s ; 0, x)$ are the solution of

$$
\dot{\Theta}_{n_{k}}(s ; 0, x)=\nabla_{\eta_{n_{k}}} v_{n_{k}}\left(\eta_{n_{k}}(s ; 0, x), s\right) \Theta_{n_{k}}(s ; 0, x) \text {, and } \Theta_{n_{k}}(0 ; 0, x)=I,
$$

and

$$
\dot{\Theta}(s ; 0, x)=\nabla_{\eta} v(\eta(s ; 0, x), s) \Theta(s ; 0, x), \text { and } \Theta(0 ; 0, x)=I,
$$

respectively.

First, we claim that there exists $\tilde{M}$ such that

$$
\left\|\Theta_{n_{k}}(s ; 0, x)\right\| \leq \tilde{M}<+\infty,\|\Theta(s ; 0, x)\| \leq \tilde{M}<+\infty .
$$

In fact, by (2.27),

$$
\Theta_{n_{k}}(s ; 0, x)=I+\int_{0}^{s} \nabla_{\eta_{n_{k}}} v_{n_{k}}\left(\eta_{n_{k}}(s ; 0, x), s\right) \Theta_{n_{k}}(s ; 0, x) d s .
$$

Hence,

$$
\left\|\Theta_{n_{k}}(s ; 0, x)\right\| \leq\|I\|+\int_{0}^{s}\left\|\nabla_{\eta_{n_{k}}} v_{n_{k}}\left(\eta_{n_{k}}(s ; 0, x), s\right)\right\|\left\|\Theta_{n_{k}}(s ; 0, x)\right\| d s .
$$


By the Gronwall inequality[10, Lemma 1.1], we know that

$$
\begin{aligned}
\left\|\Theta_{n_{k}}(s ; 0, x)\right\| & \leq\|I\| e^{\int_{0}^{s}\left\|\nabla_{n_{n_{k}}} v_{n_{k}}\left(\eta_{n_{k}}(s ; 0, x), s\right)\right\| d s} \leq\|I\| e^{K \int_{0}^{\tau}\left\|L v_{n_{k}}(\cdot, s)\right\|_{L^{2}(\Omega)} d s} \\
& \leq\|I\| e^{K \tau^{\frac{1}{2}}\left(\int_{0}^{\tau}\left\|L v_{n_{k}}(\cdot, s)\right\|_{L^{2}(\Omega)}^{2} d s\right)^{\frac{1}{2}}} \leq\|I\| e^{K \tau^{\frac{1}{2}} M^{\frac{1}{2}}} \doteq \tilde{M} .
\end{aligned}
$$

Similarly, we have $\|\Theta(s ; 0, x)\| \leq \tilde{M}$. This leads to the (2.29).

Next, we claim that

$$
\nabla_{x}\left[\int_{0}^{t}\left[v_{n_{k}}(x, r)-v(x, r)\right] d r\right] \stackrel{k}{\rightarrow} 0 \quad \text { uniformly in } x \in \Omega \times[0, \tau] .
$$

Let $w_{n_{k}}(x, t) \triangleq v_{n_{k}}(x, t)-v(x, t), z_{n_{k}}(x, t) \triangleq \int_{0}^{t} w_{n_{k}}(x, t) d t$. Since $v_{n_{k}}(x, t) \stackrel{k}{\longrightarrow} v(x, t)$, $w_{n_{k}}(x, t) \stackrel{k}{\longrightarrow} 0$ and $\left\|w_{n_{k}}\right\|_{\mathcal{F}} \leq 2 M$ for all $n_{k}$.

If $x, y \in \Omega$ and $s, t \in[0, \tau]$, then

$$
\begin{aligned}
& \left\|z_{n_{k}}(x, s)-z_{n_{k}}(y, t)\right\| \leq\left\|\int_{0}^{s}\left[w_{n_{k}}(x, r)-w_{n_{k}}(y, r)\right] d r\right\|+\left\|\int_{s}^{t} w_{n_{k}}(y, r) d r\right\| \\
= & \left\|\int_{0}^{s}\left\{\left[v_{n_{k}}(x, r)-v_{n_{k}}(y, r)\right]-[v(x, r)-v(y, r)]\right\} d r\right\|+\left\|\int_{s}^{t} w_{n_{k}}(y, r) d r\right\| \\
\leq & K\left\|\int_{0}^{\tau}\right\| L v_{n_{k}}(\cdot, r)\left\|_{L^{2}(\Omega)} d r\right\|\|x-y\|+K\left\|\int_{0}^{\tau}\right\| L v(\cdot, r)\left\|_{L^{2}(\Omega)} d r\right\|\|x-y\|+\left\|\int_{s}^{t} w_{n_{k}}(y, r) d r\right\| \\
\leq & K \tau^{\frac{1}{2}}\left(\int_{0}^{\tau}\left\|L v_{n_{k}}(\cdot, r)\right\|_{L^{2}(\Omega)}^{2} d r\right)^{\frac{1}{2}}\|x-y\|+K \tau^{\frac{1}{2}}\left(\int_{0}^{\tau}\|L v(\cdot, r)\|_{L^{2}(\Omega)}^{2} d r\right)^{\frac{1}{2}}\|x-y\|+\left\|\int_{s}^{t} w_{n_{k}}(y, r) d r\right\| \\
\leq & 2 K M^{\frac{1}{2}} \tau^{\frac{1}{2}}\|x-y\|+\left\|\int_{s}^{t} w_{n_{k}}(y, r) d r\right\| \leq 2 K M^{\frac{1}{2}} \tau^{\frac{1}{2}}\|x-y\|+|t-s|^{\frac{1}{2}}\left\|w_{n_{k}}(\cdot, r)\right\|_{\mathcal{F}} \\
\leq & 2 K M^{\frac{1}{2}} \tau^{\frac{1}{2}}\|x-y\|+2 M|t-s|^{\frac{1}{2}} .
\end{aligned}
$$

Hence, $\left\{z_{n_{k}}\right\} \subset C(\Omega \times[0, \tau])^{3}$ is equicontinuous, and the Arzela-Ascoli Theorem[12 implies that $\left\{z_{n_{k}}\right\}$ is relative compact in $C(\Omega \times[0, \tau])^{3}$, that is, any bounded sequence has a convergent subsequence in $C(\Omega \times[0, \tau])^{3}$.

Since $w_{n_{k}} \stackrel{k}{\rightarrow} 0$ weakly in $\mathcal{F}$, we know $z_{n_{k}} \stackrel{k}{\rightarrow} 0$ by contradiction(cf. [5]). That implies,

$$
\int_{0}^{t}\left[v_{n_{k}}(x, r)-v(x, r)\right] d r \stackrel{k}{\rightarrow} 0 \text { uniformly in } \Omega \times[0, \tau] .
$$


Similarly,

$$
\begin{aligned}
& \left\|\nabla_{x} z_{n_{k}}(x, s)-\nabla_{y} z_{n_{k}}(y, t)\right\| \leq\left\|\int_{0}^{s}\left[\nabla_{x} w_{n_{k}}(x, r)-\nabla_{y} w_{n_{k}}(y, r)\right] d r\right\|+\left\|\int_{s}^{t} \nabla_{y} w_{n_{k}}(y, r) d r\right\| \\
= & \left\|\int_{0}^{s}\left[\nabla_{x} v_{n_{k}}(x, r)-\nabla_{y} v_{n_{k}}(y, r)\right]-\left[\nabla_{x} v(x, r)-\nabla_{y} v(y, r)\right] d r\right\|+\left\|\int_{s}^{t} \nabla_{y} w_{n_{k}}(y, r) d r\right\| \\
\leq & K \int_{0}^{\tau}\left\|L v_{n_{k}}(\cdot, r)\right\|_{L^{2}(\Omega)} d r\|x-y\|^{\frac{1}{2}}+K \int_{0}^{\tau}\|L v(\cdot, r)\|_{L^{2}(\Omega)} d r\|x-y\|^{\frac{1}{2}}+\left\|\int_{s}^{t} \nabla_{y} w_{n_{k}}(y, r) d r\right\| \\
\leq & K \tau^{\frac{1}{2}}\left(\int_{0}^{\tau}\left\|L v_{n_{k}}(\cdot, r)\right\|_{L^{2}(\Omega)}^{2} d r\right)^{\frac{1}{2}}\|x-y\|^{\frac{1}{2}}+K \tau^{\frac{1}{2}}\left(\int_{0}^{\tau}\|L v(\cdot, r)\|_{L^{2}(\Omega)}^{2} d r\right)^{\frac{1}{2}}\|x-y\|^{\frac{1}{2}}+\left\|\int_{s}^{t} \nabla_{y} w_{n_{k}}(y, r) d r\right\| \\
\leq & 2 K M^{\frac{1}{2}} \tau^{\frac{1}{2}}\|x-y\|^{\frac{1}{2}}+\left\|\int_{s}^{t} \nabla_{y} w_{n_{k}}(y, r) d r\right\| \leq 2 K M^{\frac{1}{2}} \tau^{\frac{1}{2}}\|x-y\|^{\frac{1}{2}}+|t-s|^{\frac{1}{2}} K\left\|w_{n_{k}}(\cdot, r)\right\|_{\mathcal{F}} \\
\leq & 2 K M^{\frac{1}{2}} \tau^{\frac{1}{2}}\|x-y\|^{\frac{1}{2}}+2 M K|t-s|^{\frac{1}{2}} .
\end{aligned}
$$

Hence, $\left\{\nabla_{x} z_{n_{k}}(x, s)\right\}$ is also relative compact in $C(\Omega \times[0, \tau])^{3}$.

Passing to a subsequence, we may assume that $\nabla_{x} z_{n_{k}}(x, s) \stackrel{k}{\rightarrow} G(x)$. Then $\nabla_{x} z_{n_{k}}(x, s) \stackrel{k}{\rightarrow}$ $G(x)$ uniformly on $\Omega \times[0, \tau]$. On the other hand, (2.32) implies that $z_{n_{k}}(x, s) \stackrel{k}{\rightarrow} 0$ uniformly on $\Omega \times[0, \tau]$. Then we have $G(x)=\nabla_{x} 0=O_{3 \times 3}$. Therefore, $\nabla_{x} z_{n_{k}}(x, s) \stackrel{k}{\rightarrow} O_{3 \times 3}$ uniformly in $\Omega \times[0, \tau]$, i.e., (2.31) holds.

Finally, we turn to showing that

$$
\Theta_{n_{k}}(s ; 0, x) \stackrel{k}{\rightarrow} \Theta(s ; 0, x) .
$$

By Lemma 2.3, we obtain that

$$
\begin{aligned}
\Theta_{n_{k}}(s ; 0, x)-\Theta(s ; 0, x) & =\int_{0}^{s} \nabla_{\eta_{n_{k}}} v_{n_{k}}\left(\eta_{n_{k}}(s ; 0, x), s\right) \Theta_{n_{k}}(s ; 0, x) d s \\
& -\int_{0}^{s} \nabla_{\eta} v(\eta(s ; 0, x), s) \Theta(s ; 0, x) d s .
\end{aligned}
$$

Then by Lemma 2.1] we have

$$
\begin{aligned}
& \left\|\Theta_{n_{k}}(s ; 0, x)-\Theta(s ; 0, x)\right\| \\
& \leq \int_{0}^{s}\left\|\left[\nabla_{\eta_{n_{k}}} v_{n_{k}}\left(\eta_{n_{k}}(s ; 0, x), s\right)-\nabla_{\eta} v_{n_{k}}(\eta(s ; 0, x), s)\right] \Theta_{n_{k}}(s ; 0, x)\right\| d s \\
& +\int_{0}^{s}\left\|\nabla_{\eta} v_{n_{k}}(\eta(s ; 0, x), s)\left[\Theta_{n_{k}}(s ; 0, x)-\Theta(s ; 0, x)\right]\right\| d s \\
& +\left\|\int_{0}^{s}\left[\nabla_{\eta} v_{n_{k}}(\eta(s ; 0, x), s)-\nabla_{\eta} v(\eta(s ; 0, x), s)\right] \Theta(s ; 0, x) d s\right\| \\
& =I_{1}+I_{2}+I_{3} .
\end{aligned}
$$

By Lemma 2.1, we have

$$
\begin{aligned}
& I_{1} \leq \int_{0}^{s} K\left\|L v_{n_{k}}(\cdot, s)\right\|_{L^{2}(\Omega)}\left\|\eta_{n_{k}}(s ; 0, x)-\eta(s ; 0, x)\right\|^{\frac{1}{2}}\left\|\Theta_{n_{k}}(s ; 0, x)\right\| d s \\
& \leq K \tilde{M} \tau^{\frac{1}{2}}\left(\int_{0}^{s}\left\|L v_{n_{k}}(\cdot, s)\right\|_{L^{2}(\Omega)}^{2} d s\right)^{\frac{1}{2}}\left\|\eta_{n_{k}}(s ; 0, x)-\eta(s ; 0, x)\right\|_{C([0, \tau]: \bar{\Omega})}^{\frac{1}{2}} \\
& \leq K \tilde{M} \tau^{\frac{1}{2}} M^{\frac{1}{2}}\left\|\eta_{n_{k}}(s ; 0, x)-\eta(s ; 0, x)\right\|_{C([0, \tau]: \bar{\Omega})}^{\frac{1}{2}},
\end{aligned}
$$




$$
\begin{aligned}
I_{2} & \leq\left\|\int_{0}^{s}\right\| \nabla_{\eta} v_{n_{k}}(\eta(s ; 0, x), s)\|\| \Theta_{n_{k}}(s ; 0, x)-\Theta(s ; 0, x)\|d s\| \\
& \leq \int_{0}^{s}\left\|\nabla_{\eta} v_{n_{k}}(\eta(s ; 0, x), s)\right\|\left\|\Theta_{n_{k}}(s ; 0, x)-\Theta(s ; 0, x)\right\| d s,
\end{aligned}
$$

On the other hand, we know $\left\|\eta_{n_{k}}(s ; 0, x)-\eta(s ; 0, x)\right\|_{C([0, \tau]: \bar{\Omega})} \stackrel{k}{\rightarrow} 0$ by (ii). This leads to $I_{1} \rightarrow 0$ by (2.36). By (2.31), we know that $\nabla_{x}\left[\int_{0}^{\tau} v_{n_{k}}(x, r)-v(x, r) d r\right] \stackrel{k}{\rightarrow} O_{3 \times 3}$ uniformly on $\Omega$. This implies that $I_{3} \rightarrow 0$.

Hence, $I_{1}+I_{3} \rightarrow 0$ as $n_{k} \rightarrow \infty$. Then there exist $N=N(\varepsilon)$, such that $I_{1}+I_{3}<\varepsilon$ as $n_{k}>N$. Therefore, it follows from (2.35) that

$$
\left\|\Theta_{n_{k}}(s ; 0, x)-\Theta(s ; 0, x)\right\| \leq \varepsilon+\int_{0}^{s}\left\|\nabla_{\eta} v_{n_{k}}(\eta(s ; 0, x), s)\right\|\left\|\Theta_{n_{k}}(s ; 0, x)-\Theta(s ; 0, x)\right\| d s .
$$

and Gronwall inequality[10, Lemma 1.1] implies that

$$
\left\|\Theta_{n_{k}}(s ; 0, x)-\Theta(s ; 0, x)\right\| \leq \varepsilon e^{\int_{0}^{s}\left\|\nabla_{\eta} v_{n_{k}}(\eta(s ; 0, x), s)\right\| d s} \leq \varepsilon e^{K \tau^{\frac{1}{2}}\left(\int_{0}^{\tau}\left\|v_{n_{k}}(\cdot, s)\right\|_{L}^{2} d s\right)^{\frac{1}{2}}} \leq \varepsilon e^{K \tau^{\frac{1}{2}} M^{\frac{1}{2}}} .
$$

So (2.33) is proved by letting $\varepsilon \rightarrow 0$.

(iv) For the sake of simplicity, in what follows, we denote $\Theta_{n_{k}}(s ; 0, x)$ and $\Theta(s ; 0, x)$ by $\Theta_{n_{k}}$ and $\Theta$, respectively. Then,

$$
\begin{aligned}
\left\|\Theta_{n_{k}} \Theta_{n_{k}}^{T}-\Theta \Theta^{T}\right\| & \leq\left\|\Theta_{n_{k}} \Theta_{n_{k}}^{T}-\Theta \Theta_{n_{k}}^{T}\right\|+\|\Theta\|\left\|\Theta_{n_{k}}^{T}-\Theta \Theta^{T}\right\| \\
& \leq\left\|\Theta_{n_{k}}-\Theta\right\|\left\|\Theta_{n_{k}}^{T}\right\|+\|\Theta\|\left\|\Theta_{n_{k}}^{T}-\Theta^{T}\right\| \\
& \leq \tilde{M}\left\|\Theta_{n_{k}}-\Theta\right\|+\tilde{M}\left\|\Theta_{n_{k}}^{T}-\Theta^{T}\right\| \stackrel{k}{\rightarrow} 0,
\end{aligned}
$$

since $\Theta_{n_{k}} \stackrel{k}{\rightarrow} \Theta$ and $\Theta_{n_{k}}^{T} \stackrel{k}{\rightarrow} \Theta^{T}$ by part (iii).

Similarly, there holds $\left\|\Theta_{n_{k}}^{T} \Theta_{n_{k}}-\Theta^{T} \Theta\right\| \stackrel{k}{\rightarrow} 0$.

Now we prove that

$$
R_{n_{k}} \stackrel{k}{\rightarrow} R
$$

Let $A_{n_{k}}=\Theta_{n_{k}}(s ; 0, x) \Theta_{n_{k}}^{T}(s ; 0, x)=\left(a_{i j}^{n_{k}}(s ; 0, x)\right)_{3 \times 3}, A=\Theta(s ; 0, x) \Theta^{T}(s ; 0, x)=\left(a_{i j}(s ; 0, x)\right)_{3 \times 3}$.

By (2.38), we know $a_{i j}^{n_{k}}(s ; 0, x) \stackrel{k}{\rightarrow} a_{i j}(s ; 0, x)$ for $i, j=1,2,3$. Now, we simply denote $a_{i j}^{n_{k}}(s ; 0, x)$ and $a_{i j}(s ; 0, x)$ by $a_{i j}^{n_{k}}$ and $a_{i j}$, respectively.

By Lemma 2.3, we obtain that

$$
\operatorname{det}\left(\Theta_{n_{k}}(\tau ; 0, x)\right)=e^{-\int_{0}^{\tau} \sum_{i=1}^{3}\left(v_{n_{k}}\left(\eta_{n_{k}}(s ; t, x), s\right)\right)_{i, x_{i}} d s} \neq 0 .
$$

By (2.40), we know that $\Theta_{n_{k}} \Theta_{n_{k}}^{T}, \Theta \Theta^{T} \in S P D(3)$. Let $\lambda_{n_{k}}^{(1)} \geq \lambda_{n_{k}}^{(2)} \geq \lambda_{n_{k}}^{(3)}>0, \lambda^{(1)} \geq$ $\lambda^{(2)} \geq \lambda^{(3)}>0$ be the eigenvalues of $\Theta_{n_{k}} \Theta_{n_{k}}^{T}, \Theta \Theta^{T}$ respectively. By Lemma 2.4, we obtain $\lambda_{n_{k}}^{(i)} \rightarrow \lambda^{(i)}(i=1,2,3)$. 
By (2.40), we obtain that

$$
\begin{aligned}
\operatorname{det}\left(A_{n_{k}}\right) & =e^{-2 \int_{0}^{\tau} \sum_{i=1}^{3}\left(v_{n_{k}}\left(\eta_{n_{k}}(s ; t, x), s\right)\right)_{i, x_{i}} d s} \leq e^{2 \int_{0}^{\tau}\|\nabla v(\cdot, s)\| d s} \leq e^{2 \int_{0}^{\tau}\left\|v_{n_{k}}(\cdot, s)\right\|_{\left[C^{1, \frac{1}{2}}(\Omega)\right]^{3}} d s} \\
& \leq e^{2 C \int_{0}^{\tau}\left\|v_{n_{k}}(\cdot, s)\right\|_{\left[H_{0}^{3}(\Omega)\right]^{3}} d s} \leq e^{2 K \int_{0}^{\tau}\left\|L v_{n_{k}}(\cdot, s)\right\|_{L^{2}(\Omega)} d s} \leq e^{2 K \tau^{\frac{1}{2}}\left[\int_{0}^{\tau}\left\|L v_{n_{k}}(\cdot, s)\right\|_{L^{2}(\Omega)}^{2} d s\right]^{\frac{1}{2}}} \\
& \leq e^{2 K \tau^{\frac{1}{2}} M^{\frac{1}{2}}} \doteq M_{1}<+\infty .
\end{aligned}
$$

In a similar way, we can obtain that

$$
\operatorname{det}\left(A_{n_{k}}\right) \geq \frac{1}{M_{1}}
$$

By singularity decomposition theorem [7] (See Appendix 2), we can find two orthogonal matrix $U_{n_{k}}, V_{n_{k}}$ such that $\Theta_{n_{k}}=U_{n_{k}} S_{n_{k}} V_{n_{k}}^{T}$, where $S^{n_{k}}=\operatorname{diag}\left(\sqrt{\lambda_{n_{k}}^{(1)}}, \sqrt{\lambda_{n_{k}}^{(2)}}, \sqrt{\lambda_{n_{k}}^{(3)}}\right), \lambda_{n_{k}}^{(1)}, \lambda_{n_{k}}^{(2)}, \lambda_{n_{k}}^{(3)}$ are eigenvalues of $\Theta_{n_{k}} \Theta_{n_{k}}^{T}, U_{n_{k}}, V_{n_{k}}$ are orthogonal eigenvectors of $\Theta_{n_{k}} \Theta_{n_{k}}^{T}$ and $\Theta_{n_{k}}^{T} \Theta_{n_{k}}$ respectively.

Then, $A_{n_{k}}=\Theta_{n_{k}} \Theta_{n_{k}}^{T}=U_{n_{k}}\left(S_{n_{k}}\right)^{2} U_{n_{k}}^{T}$ and $\left(A_{n_{k}}\right)^{-1}=U_{n_{k}}\left(S_{n_{k}}\right)^{-2} U_{n_{k}}^{T}$. Hence,

$$
\begin{aligned}
\left\|\left(A_{n_{k}}\right)^{-1}\right\| & \leq\left\|U_{n_{k}}\right\|\left\|\left(S_{n_{k}}\right)^{-2}\right\|\left\|U_{n_{k}}^{T}\right\| \leq\left\|U_{n_{k}}\right\|^{2}\left[\frac{1}{\lambda_{n_{k}}^{(1)}}+\frac{1}{\lambda_{n_{k}}^{(2)}}+\frac{1}{\lambda_{n_{k}}^{(3)}}\right] \\
& \leq\left\|U_{n_{k}}\right\|^{2} \frac{\lambda_{n_{k}}^{(1)} \lambda_{n_{k}}^{(2)}+\lambda_{n_{k}}^{(1)} \lambda_{n_{k}}^{(3)}+\lambda_{n_{k}}^{(2)} \lambda_{n_{k}}^{(3)}}{\lambda_{n_{k}}^{(1)} \lambda_{n_{k}}^{(2)} \lambda_{n_{k}}^{(3)}}=\left\|U_{n_{k}}\right\|^{2} \frac{\lambda_{n_{k}}^{(1)} \lambda_{n_{k}}^{(2)}+\lambda_{n_{k}}^{(1)} \lambda_{n_{k}}^{(3)}+\lambda_{n_{k}}^{(2)} \lambda_{n_{k}}^{(3)}}{\operatorname{det}\left(A_{n_{k}}\right)} \\
& \leq\left\|U_{n_{k}}\right\|^{2} \frac{\left[\lambda_{n_{k}}^{(1)}+\lambda_{n_{k}}^{(2)}+\lambda_{n_{k}}^{(3)}\right]^{2}}{\operatorname{det}\left(A_{n_{k}}\right)}=\left\|U_{n_{k}}\right\|^{2} \frac{\left[\operatorname{tr}\left(A_{n_{k}}\right)\right]^{2}}{\operatorname{det}\left(A_{n_{k}}\right)} \leq\left\|U_{n_{k}}\right\|^{2} \frac{\left\|A_{n_{k}}\right\|^{2}}{\operatorname{det}\left(A_{n_{k}}\right)} \\
& \leq 27 \tilde{M}^{2} M_{1} \doteq M_{2}<+\infty
\end{aligned}
$$

by (2.41), (2.42) and (2.30), where $\operatorname{tr}(A)$ denote the trace of matrix $A$.

Similarly, we know that $\left\|A^{-1}\right\| \leq M_{2}$.

By (2.43), we obtain that

$$
\left\|\left(A_{n_{k}}\right)^{-1}-A^{-1}\right\|=\left\|A_{n_{k}}^{-1}\left(A-A_{n_{k}}\right) A^{-1}\right\| \leq\left\|A_{n_{k}}^{-1}\right\|\left\|A-A_{n_{k}}\right\|\left\|A^{-1}\right\| \stackrel{k}{\rightarrow} 0,
$$

since $A-A_{n_{k}}=\Theta_{n_{k}} \Theta_{n_{k}}^{T}-\Theta \Theta^{T} \rightarrow 0$ by (2.38).

Hence, $A_{n_{k}}^{-1} \stackrel{k}{\rightarrow} A^{-1}$.

Since $A_{n_{k}}^{-1}=U_{n_{k}}\left(S_{n_{k}}\right)^{-2} U_{n_{k}}^{T}=\left[U_{n_{k}} S_{n_{k}}^{-1} U_{n_{k}}^{T}\right]\left[U_{n_{k}} S_{n_{k}}^{-1} U_{n_{k}}^{T}\right] \triangleq B_{n_{k}} B_{n_{k}}, A^{-1}=U S^{-2} U^{T}=$ $\left[U S^{-1} U^{T}\right]\left[U S^{-1} U^{T}\right] \triangleq B B$, then $B_{n_{k}}$ and $B$ are positive definite symmetric matrixes. By Lemma 2.4, we know $\operatorname{det}\left(B_{n_{k}}\right)=\frac{1}{\sqrt{\lambda_{n_{k}}^{(1)} \lambda_{n_{k}}^{(2)} \lambda_{n_{k}}^{(3)}}} \rightarrow \frac{1}{\sqrt{\lambda^{(1)} \lambda^{(2)} \lambda^{(3)}}}=\operatorname{det}(B)$. Since $B_{n_{k}}, B \in$ $S P D(3)$, the Minkowskii inequality implies

$$
\left[\operatorname{det}\left(B_{n_{k}}+B\right)\right]^{\frac{1}{3}} \geq\left[\operatorname{det}\left(B_{n_{k}}\right)\right]^{\frac{1}{3}}+[\operatorname{det}(B)]^{\frac{1}{3}} \geq[\operatorname{det}(B)]^{\frac{1}{3}} .
$$

This is, $\operatorname{det}\left(B_{n_{k}}+B\right) \geq \operatorname{det}(B)=\frac{1}{\lambda^{(1)} \lambda^{(2)} \lambda^{(3)}}>0$. 
Further more, we have

$$
\begin{aligned}
& \left\|B_{n_{k}}+B\right\| \leq\left\|B_{n_{k}}\right\|+\|B\| \leq\left\|U_{n_{k}}\right\|^{2}\left[\frac{1}{\sqrt{\lambda_{n_{k}}^{(1)}}}+\frac{1}{\sqrt{\lambda_{n_{k}}^{(2)}}}+\frac{1}{\sqrt{\lambda_{n_{k}}^{(3)}}}\right]+\|U\|^{2}\left[\frac{1}{\sqrt{\lambda^{(1)}}}+\frac{1}{\sqrt{\lambda^{(2)}}}+\frac{1}{\sqrt{\lambda^{(3)}}}\right] \\
& =\left\|U_{n_{k}}\right\|^{2} \frac{\sqrt{\lambda_{n_{k}}^{(1)} \lambda_{n_{k}}^{(2)}}+\sqrt{\lambda_{n_{k}}^{(1)} \lambda_{n_{k}}^{(3)}}+\sqrt{\lambda_{n_{k}}^{(2)} \lambda_{n_{k}}^{(3)}}}{\sqrt{\lambda_{n_{k}}^{(1)} \lambda_{n_{k}}^{(2)} \lambda_{n_{k}}^{(3)}}}+\|U\|^{2} \frac{\sqrt{\lambda^{(1)} \lambda^{(2)}}+\sqrt{\lambda^{(1)} \lambda^{(3)}}+\sqrt{\lambda^{(2)} \lambda^{(3)}}}{\sqrt{\lambda^{(1)} \lambda^{(2)} \lambda^{(3)}}} \\
& \leq\left\|U_{n_{k}}\right\|^{2} \frac{\lambda_{n_{k}}^{(1)}+\lambda_{n_{k}}^{(2)}+\lambda_{n_{k}}^{(3)}}{\sqrt{\lambda_{n_{k}}^{(1)} \lambda_{n_{k}}^{(2)} \lambda_{n_{k}}^{(3)}}}+\|U\|^{2} \frac{\lambda^{(1)}+\lambda^{(2)}+\lambda^{(3)}}{\sqrt{\lambda^{(1)} \lambda^{(2)} \lambda^{(3)}}}=\left\|U_{n_{k}}\right\|^{2} \frac{\operatorname{tr}\left(A_{n_{k}}\right)}{\sqrt{\operatorname{det}\left(A_{n_{k}}\right)}}+\|U\|^{2} \frac{\operatorname{tr}(A)}{\sqrt{\operatorname{det}(A)}} \\
& \leq 9 M_{2} \sqrt{M_{1}}+9 M_{2} \sqrt{M_{1}} \leq 18 M_{2} \sqrt{M_{1}} \doteq M_{3}<+\infty \text {. }
\end{aligned}
$$

By (2.44), we obtain that

$$
A_{n_{k}}^{-1}-A^{-1}=B_{n_{k}}^{2}-B^{2}=\left(B_{n_{k}}+B\right)\left(B_{n_{k}}-B\right) \stackrel{k}{\rightarrow} 0 .
$$

By (2.47) and Lemma 2.5, we obtain that $\left(\Theta_{n_{k}} \Theta_{n_{k}}^{T}\right)^{-\frac{1}{2}}=B_{n_{k}} \rightarrow B=\left(\Theta \Theta^{T}\right)^{-\frac{1}{2}}$.

Based on the above calculation, we obtain that

$$
\begin{aligned}
& \left\|\Theta_{n_{k}}\left(\Theta_{n_{k}} \Theta_{n_{k}}^{T}\right)^{-\frac{1}{2}}-\Theta(\Theta \Theta)^{-\frac{1}{2}}\right\| \\
\leq & \left\|\Theta_{n_{k}}\right\|\left\|\left(\Theta_{n_{k}} \Theta_{n_{k}}^{T}\right)^{-\frac{1}{2}}-(\Theta \Theta)^{-\frac{1}{2}}\right\|+\left\|\Theta_{n_{k}}-\Theta\right\|\left\|(\Theta \Theta)^{-\frac{1}{2}}\right\| \stackrel{k}{\rightarrow} 0 .
\end{aligned}
$$

So, $R_{n_{k}} \stackrel{k}{\rightarrow} R$.

\section{Proof of Theorem 1.1}

The aim of this section is to prove Theorem 1.1 For this purpose, we let

$$
\inf _{v \in \mathcal{F}} H(v)=\bar{H} .
$$

Proof of Theorem 1.1; Let $\left\{v_{n}\right\} \subset \mathcal{F}$ be a minimizing sequence of $H(v)$, that is,

$$
\lim _{n \rightarrow \infty} H\left(v_{n}\right)=\bar{H} .
$$

Then, there exists a constat $M>0$ such that $\left\|v_{n}\right\|_{\mathcal{F}}^{2} \leq M$, otherwise, there is a contradiction by $\lim _{\|v\|_{\mathcal{F}} \rightarrow \infty} H(v)=+\infty$.

It follows from Lemma 2.6. (i) that there exists a weakly convergent subsequence $\left\{v_{n_{k}}\right\}$ of $\left\{v_{n}\right\}$ such that

$$
v_{n_{k}} \stackrel{k}{\rightarrow} \hat{v}, \quad \text { for some } \hat{v} \in \mathcal{F} \text {. }
$$

Now, we claim that

$$
\lim _{n_{k} \rightarrow \infty} \inf H\left(v_{n_{k}}\right) \geq H(\hat{v}) .
$$


By Lemma 2.6(i), we know that

$$
M \geq \lim _{n_{k} \rightarrow \infty} \inf \int_{0}^{\tau}\left\|L v_{n_{k}}(\cdot, t)\right\|_{L^{2}(\Omega)}^{2} d t \geq \int_{0}^{\tau}\|L \hat{v}(\cdot, t)\|_{L^{2}(\Omega)}^{2} d t,
$$

Further more, by Lemma 2.6 (ii), (iii), (iv) and definition of $h_{n_{k}}(x)$ and $h(x)$, we have

$$
h_{n_{k}}(x)=\eta_{n_{k}}(0 ; \tau, x) \stackrel{k}{\rightarrow} \eta(0 ; \tau, x)=h(x) \text { for } \forall x \in \Omega,
$$

and

$$
R_{n_{k}} \stackrel{k}{\rightarrow} R .
$$

It follows from (3.6) and (3.7) that

$$
\begin{aligned}
\left\|T \diamond h_{n_{k}}(x)-T \diamond h(x)\right\| & =\left\|R_{n_{k}}\left[T \circ h_{n_{k}}(x)\right] R_{n_{k}}^{T}-R[T \circ h(x)] R^{T}\right\| \\
& \leq\left\|R_{n_{k}}\left[T \circ h_{n_{k}}(x)\right] R_{n_{k}}^{T}-R\left[T \circ h_{n_{k}}(x)\right] R_{n_{k}}^{T}\right\| \\
& +\left\|R\left[T \circ h_{n_{k}}(x)\right] R_{n_{k}}^{T}-R[T \circ h(x)] R_{n_{k}}^{T}\right\| \\
& +\left\|R[T \circ h(x)] R_{n_{k}}^{T}-R[T \circ h(x)] R^{T}\right\| \\
& \leq\left\|R_{n_{k}}-R\right\|\left\|\left[T \circ h_{n_{k}}(x)\right] R_{n_{k}}^{T}\right\| \\
& +\|R\|\left\|\left[T \circ h_{n_{k}}(x)\right]-[T \circ h(x)]\right\|\left\|R_{n_{k}}^{T}\right\| \\
& +\|R[T \circ h(x)]\|\left\|R_{n_{k}}^{T}-R^{T}\right\| \stackrel{k}{\rightarrow} 0 \text { on } x \in \Omega \backslash h^{-1}\left(\Delta_{T}\right) .
\end{aligned}
$$

Hence, $\left\|T \diamond h_{n_{k}}(\cdot)-D(\cdot)\right\|^{2} \stackrel{k}{\rightarrow}\|T \diamond h(\cdot)-D(\cdot)\|^{2}$ on $x \in \Omega \backslash h^{-1}\left(\Delta_{T}\right)$.

By Remark 2.1. we know that $h: \Omega \rightarrow \Omega$ is a 1-to-1 and onto, which ensure the existence of $h^{-1}(x)$. Therefore, $h^{-1}\left(\Delta_{T}\right)$ is a set of Lebesgue measure zero.

On the other hand, we know

$$
\left\|T \diamond h_{n_{k}}(\cdot)-D(\cdot)\right\|^{2} \leq \max _{x \in \Omega}\|T(x)-D(x)\|^{2}=J \in L^{1}(\Omega) .
$$

By the Lebesgue Dominant Convergence Theorem, we obtain that

$$
\left\|T \diamond h_{n_{k}}(\cdot)-D(\cdot)\right\|_{L^{2}(\Omega)}^{2} \stackrel{k}{\rightarrow}\|T \diamond h(\cdot)-D(\cdot)\|_{L^{2}(\Omega)}^{2} .
$$

Therefore, (3.9) and (3.5) implies that (3.4) holds.

Since $\hat{v} \in \mathcal{F}$,

$$
H(\hat{v}) \geq \inf _{v \in \mathcal{F}} H(v)=\bar{H}
$$

Combining (3.2) and (3.4), we see that

$$
\bar{H}=\lim _{n_{k} \rightarrow \infty} \inf H\left(v_{n_{k}}\right) \geq H(\hat{v}) \geq \bar{H} .
$$

That is,

$$
H(\hat{v})=\bar{H}=\inf _{v \in \mathcal{F}} H(v)
$$


For the above minimizer $\hat{v} \in \mathcal{F}$, by Lemma 2.2, we know that there exists an unique $\hat{\eta}(s ; t, x)$ such that

$$
\frac{d \hat{\eta}(s ; t, x)}{d s}=\hat{v}(\hat{\eta}(s ; t, x), s), \quad \hat{\eta}(t ; t, x)=x
$$

Hence, the mapping $\hat{h}(x)=\hat{\eta}(0 ; \tau, x): \Omega \rightarrow \Omega$ is what we want. Moreover, by (ii) in Lemma 2.6. we know $\hat{h}(x) \in\left[C^{1, \frac{1}{2}}(\Omega)\right]^{3}$ with the derivative satisfies (2.6).

Acknowledgements. This work was supported by NSFC under grant 11471331 and National Center for Mathematics and Interdisciplinary Sciences.

\section{Appendix: The calculation of $\left(\mathrm{AA}^{T}\right)^{-\frac{1}{2}}$}

By singularity decomposition theorem[7], for any matrix $\mathbf{A} \in \mathbb{R}^{n \times n}$, it could be broken down into the product of three matrixes:

$$
\mathbf{A}=\mathbf{U S V}^{T}
$$

where $\mathbf{U}^{T} \mathbf{U}=\mathbf{U U}^{T}=\mathbf{I}, \mathbf{V}^{T} \mathbf{V}=\mathbf{V} \mathbf{V}^{T}=\mathbf{I}$; The columns of $\mathbf{U}$ are orthogonal eigenvectors $[7]$ of $\mathbf{A} \mathbf{A}^{T}$, the columns of $\mathbf{V}$ are orthogonal eigenvectors of $\mathbf{A}^{T} \mathbf{A} . \mathbf{S}=\operatorname{diag}\left(\sigma^{(1)}, \sigma^{(2)}, \cdots, \sigma^{(n)}\right)$, and $\sigma^{(i)}=\sqrt{\lambda^{(i)}}(i=1,2, \cdots, n)$. Where $\lambda^{(i)}(i=1,2, \cdots, n)$ are eigenvalues of $\mathbf{A}^{T} \mathbf{A}$. Here we call $\mathbf{U}, \mathbf{V}$ orthogonal matrixes of $\mathbf{A} \mathbf{A}^{T}$ and $\mathbf{A}^{T} \mathbf{A}$.

Based on the theory mentioned above, we focus on the calculation of $\left(\mathbf{A} \mathbf{A}^{T}\right)^{-\frac{1}{2}}$.

By (.14), we obtain

$$
\mathbf{A} \mathbf{A}^{T}=\mathbf{U S V}^{T} \mathbf{V S U}^{T}=\mathbf{U S}^{2} \mathbf{U}^{T}=\mathbf{U S U}^{T} \mathbf{U S U}^{T} .
$$

Therefore, by the uniqueness of square root of matrix in [14], we obtain that

$$
\left(\mathbf{A A}^{T}\right)^{\frac{1}{2}}=\mathbf{U S U}^{T} .
$$

By (.16), we know

$$
\left(\mathbf{A A}^{T}\right)^{-\frac{1}{2}}=\mathbf{U S}^{-1} \mathbf{U}^{T}
$$

\section{References}

[1] Alexander D C, Pierpaoli C, Basser P J, Gee J C. Spatial transformations of diffusion tensor magnetic resonance images. IEEE Transaction on Medical imaging, 2001, 20: $1131-1139$

[2] Amit Y. A nonlinear vartional problem for image matching. SIAM Journal on scientific computing, 1994, 15(1): 207-224

[3] Björck A. Numerics of Gram-Schmidt orthogonalization. Linear algebra and its applications, 1994, 198: 297-316

[4] Budhiraja A, Dupuis P, Maroulas V. Large deviations for stochastic flows of diffeomorphisms. Bernoulli, 2010, 16(1): 234-257

[5] Dupuis P, Grenander U, Miller M I. Variational problems on flows of diffeomorphisms for image matching. Quarterly of Applied Mathematics, 1998, 6: 587-600 
[6] Evans L C. Partial differential equations. American Mathematical Society, Berkeley, 1997

[7] Johnson C R, Okubo K, Reams R. Uniqueness of matrix square roots and application. Linear Algebra and it applications, 2001, 323: 51-60

[8] Li J, Shi Y, Tran G, Dinov I, Wang D JJ, Toga A. Fast local trust region for diffusion tensor registration using exact reorientation and regularization. IEEE Tans Med Imaging, 2014, 33(5): 1-43

[9] Li R, Zhong S, Swartz C. An improvement of the Arzela-Ascoli theorem. Topology and its applications, 2012, 159(8): 2058-2061

[10] Ogvntuase J A. On an inequality of gronwall. Journal of Ineualities in pure and Applied Mathematics, 2001, 2(1): 1-6

[11] Strang G. Introduction to linear algebra(Forth edition). Wellesley-Cambridge Press and SIAM, 2009

[12] Teschl G. Ordinary differential equations and Dynamical systems. American Mathematical Society, 2012 